\title{
Underwater LiDAR Signal Processing for Enhanced Detection and Localization of Marine Life
}

\author{
Elizaveta Dubrovinskaya ${ }^{*}$, Fraser Dalgleish ${ }^{\S}$, Bing Ouyang ${ }^{\S}$, Paolo Casari* \\ *IMDEA Networks Institute, Madrid, Spain $\quad \ddagger$ Universidad Carlos III de Madrid, Spain \\ ${ }^{\S}$ Florida Atlantic University Harbor Branch Oceanographic Institute, Fort Pierce, FL, USA
}

\begin{abstract}
The in situ detection, recognition and tracking of marine animal species is a very important step of field research in the domains of, among others, biology and ecology. Still, the direct observation of marine wildlife through equipment operating in the visible light spectrum is often impaired by the challenging conditions offered by ocean waters, where light can be subject to scattering and attenuation phenomena due to the water turbidity. As the use of powerful lighting may prove ineffective and even induce behavioral changes in marine animals, the design of minimally or non-invasive observation instruments becomes particularly important.

In this paper, we consider the serial Light Detection And Ranging (LiDAR) system under development at the Florida Atlantic University (FAU). This LiDAR design is based on inexpensive components and on low average power red lasers which are subject to significant attenuation in water, but are both eye-safe and invisible to marine life. Considering the challenge of detecting and evaluating the presence of marine wildlife, we present a full processing pipeline for LiDAR data, that includes water turbidity detection, non-gated backscattering compensation, contrast enhancement, and the construction of a three-dimensional model of the detected target. The pipeline is applied to a number of tank test data, under different turbidity conditions.
\end{abstract}

\section{INTRODUCTION AND RELATED WORK}

Detecting and tracking marine wildlife in its natural habitat is of interest for many fields of science, including behavioral ecology and sociobiology, and can help explain their social dynamics as well as measure the impact of human interference [1]. A specific, important application is the prevention of harmful interactions with various types of humanmade underwater equipment, such as marine hydro-kinetic devices [2]. However due to the challenging characteristics of the underwater environment, such as insufficient illumination, attenuation and scattering, it may be arduous to observe marine wildlife using conventional surface approaches, such as optical surveillance in the visible light spectrum. Extra lighting does not solve the issue, as it creates flares, and may also induce behavioral changes in the animals (e.g., many species are attracted by visible light sources [3]).

Available active acoustics technologies for fisheries monitoring can be categorized as either acoustic cameras or sounders. Active acoustic cameras, such as Dual Frequency Identification Sonar (DIDSON) use an acoustic lens technology which forms images with greater detail than found in conventional sonars. While reasonably good estimates of feature dimensions can be extracted from DIDSON images, that level of detail is only available at relatively short ranges and with an angular field of view where image resolution is highest. Consequently, data bandwidth and storage become problematic. 2D imaging sonars (e.g., Teledyne BlueView products), likewise provide a video-rate output, but with a limited angular field of view and range. Acoustic profilers such as echosounders, on the other hand, are a standard instrument of fisheries hydro-acoustics. These instruments can reach a long distance (hundreds of meters) but lack the resolution and evaluation intuitiveness of traditional optical imagery [4].

Therefore, the design of non-invasive systems for marine life observation remains an interesting research problem. A possible solution is provided by marine Light Detection And Ranging (LiDARs): using a portion of the light spectrum which is invisible to most underwater species, LiDARs can image via serial laser scanning based on the received intensity of reflected light, with an additional layer of time of flight (ToF) data, which reflects the distance to the object (depth map). Therefore such systems may work without additional light sources, and due to the low average power of the emitted light, they do not affect underwater fauna [5]. In contrast to conventional optical systems, LiDARs can count on the depth map to improve the image quality, to perform accurate ranging and scaling, as well as to separate objects from one another and from the background. Beyond the single pulse per pixel type of LiDAR system that is described in this paper, modulated-pulse versions of the serial-scanning underwater LiDAR imager have been tank-tested to study the improved ranging and intensity imaging performance in turbid environments by using radar waveforms and decoding techniques [6], [7].

The low average power system thatis being developed at the Florida Atlantic University (FAU) Harbor Branch Oceanographic institution (HBOI) is called Unobtrusive Multistatic Serial LiDAR imager (UMSLI). The configuration of UMSLI described in this paper consists of one laser transmitter, and one to three receivers. It has two working modes: the detection mode, when the laser does a sparse spatial scan, and the dense mode, when the scan area is reduced to the point of detection, so that the LiDAR can produce a detailed image of the scanned underwater object. The data collected by this system is further used for fish detection, localization and identification that has to be performed on the go. For the complete description of this project, we refer the interested reader to [8]. This particular LiDAR system uses relatively inexpensive components, and its overall price is foreseen to be about one order of magnitude lower than other systems available on the market. It uses 
low average power red laser diodes with a wavelength of $638 \mathrm{~nm}$, which suffer from higher attenuation in water, but are unobtrusive for fish vision. Together with a relatively low sampling frequency, these and other aspects of UMSLI require a targeted approach to the processing of the obtained data.

The UMSLI prototype was developed and validated at the optical test facility at HBOI. The serial scanning technique on which the LiDAR design hinges has been employed in nearmonostatic [9], [10] and bi-static [11] configurations. Serial scanning can be instrumental to help detect individuals within a cluster of animals better than by using a regular camera system. Furthermore, it can enable the formation of images even when the line-of sight between the target and the detector is occluded [12].

In the following sections we provide further details about the UMSLI design and experimental setup (Section II), we describe our data processing pipeline (Section III), and provide concluding remarks in Section IV.

\section{System DESCRIPTION AND EXPERIMENT SETUP}

\section{A. Description of the LiDAR system}

The integrated system adopts a multi-tiered design that consists of the sensing hardware, of image enhancement, detection, and classification, as well as of data archiving.

The UMSLI sensing front end consists of six receivers (Rx), six transmitters (Tx), and a digital signal processor [8]. The transmitters scan a water volume in a bi-directional raster pattern. The scan field can be configured to be either sparse (with fewer pulses over a wider angle, used mainly for detection purposes), or dense (concentrating a higher pulse density through a narrower range of angles, typically after the detection of an object has been confirmed). On the one hand, the depth of field for each channel depends on the depth of the overlapping region between the laser beam and the receiver's field of view. On the other hand, the image resolution depends on the diameter of the pulsed laser beam as it intersects a hard target. The receivers consist of a high-speed photomultiplier relying on focusing optics and on a spectral bandpass filter, designed to efficiently collect time-resolved back-irradiated light from the emitted laser pulses. Depending on the conditions of the water, such light may consist of either reflections from objects or from backscattering. The signals received by the photomultipliers are digitized at a rate of one sample every $0.5 \mathrm{~ns}$, before they are conveyed to digital processing elements that reconstruct the areas of interest in the water volume.

Given its unobtrusiveness with respect to marine fauna vision and behavior, the UMSLI system can be used in the proximity of marine installations to ensure the monitoring of endangered/threatened species, and to assess the impact of the installation on marine wildlife. The image formation approach has been demonstrated to be especially suitable for turbid or low-light applications. This makes the UMSLI more convenient than traditional camera solutions, potentially achieving persistent surveillance through day and night, while still providing the operational advantages of optical solutions.

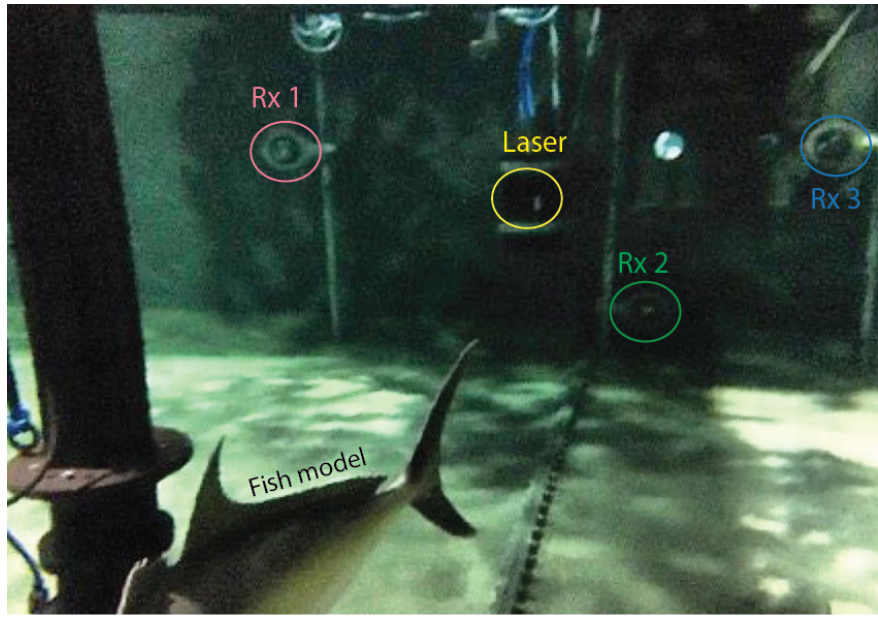

Fig. 1. Photo of the test tank environment, showing the location of the laser transmitter and of the three receivers, as well as the model fish target (in this case, an amberjack model).

\section{B. Test scenario and data sets}

In this paper, we consider two data sets from previous experiments taken in a controlled test tank environment at HBOI. One data set contains experimental results from a system with a single light emitter and a single receiver, which captured images of a static fish model in different poses, with $200 \times 200$ pulse resolution, at 128 samples per $128-n s$ pulse record. The frames are taken in clear water, as well as by controlling the turbidity such that the total attenuation coefficient at $638 \mathrm{~nm}$ ranges up to $c=0.75 \mathrm{~m}^{-1}$ (turbid water) using sparse and dense mode scans. Altogether, the data set contains 79 frames.

The second data set has a similar structure, but is obtained from another version of UMSLI with three receivers having a higher sampling resolution of 256 samples per 128-ns pulse record. A picture of the test tank with this configuration of the LiDAR is shown in Fig. 1. The laser occupies a central position, whereas the three receivers are deployed to the side of the laser and below it. Fig. 1 also shows part of the model fish target (an amberjack in this case). Seven different scenes were taken for each turbidity setting, with moving and static fish models, as well as with a calibration board. Each scene was taken 15 times, hence the data set contains altogether over 500 frames per receiver. These challenging data sets provide an interesting test platform to seek an optimal solution for the enhancement of LiDAR data under power and computational complexity constraints. The following section explains our proposed processing pipeline to produce classifier-friendly images and depth maps for further post-processing stages.

\section{LiDAR Data Processing PiPEline AND Results}

In this section, we present the steps of the LiDAR data processing pipeline. We start with water turbidity estimation in Section III-A. The following steps include image enhancement via backscattering removal (Section III-B), and contrast enhancement for 2D visualization (Section III-C). Finally, the 


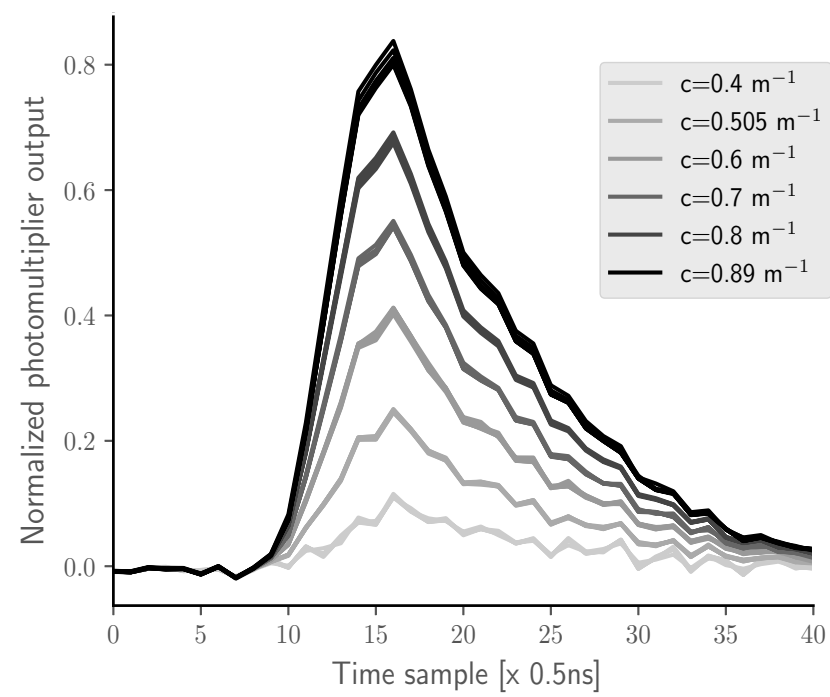

Fig. 2. Backscattered light intensity over time for different turbidity values.

LiDAR scan is transformed into a 3D cloud of points. Those that are not part of the target are identified and removed via a clustering algorithm; the remaining points are then employed to construct a 3D model of the target, which can be employed for biomass evaluation (Section III-D).

\section{A. Turbidity estimation}

An important preliminary step to achieve good-quality $\mathrm{Li}$ DAR imaging is to estimate environmental parameters, and specifically turbidity, which is expressed in terms of the total light intensity attenuation coefficient of the water $c\left[\mathrm{~m}^{-1}\right]$. In the current setup for test tank experiments, additional equipment is used for precise turbidity measurements, which is advantageous for further image processing. However, once the LiDAR is deployed at sea, it might be challenging to measure the ocean water's turbidity without such additional equipment, especially in the presence of mixing phenomena that may induce turbidity variations over time. In these cases, the backscattering intensity can be a good measure for turbidity [13]. Several parameters of the LiDAR affect backscattering. These include the wavelength, power and gain of the incident laser beam, the scan area, and the relative position of the transmitter and receiver [14]. By including these parameters in a backscattering model, the LiDAR can be calibrated to infer turbidity from backscattering measurements.

We employed the FAU test tank to carry out several backscattering calibration measurements in different water turbidity conditions. The results can be observed in Fig. 2, which presents a superposition of several backscattered light intensity signals sampled at intervals of $0.5 \mathrm{~ns}$. For the same value of $c$, the signals show approximately the same backscattering peak value. However, this peak varies significantly by varying $c$. As observed in Fig. 3, the relationship between the backscattering intensity peak value and the turbidity level expressed through $c$ is roughly linear. A least-squares fit (top panel in Fig. 3)

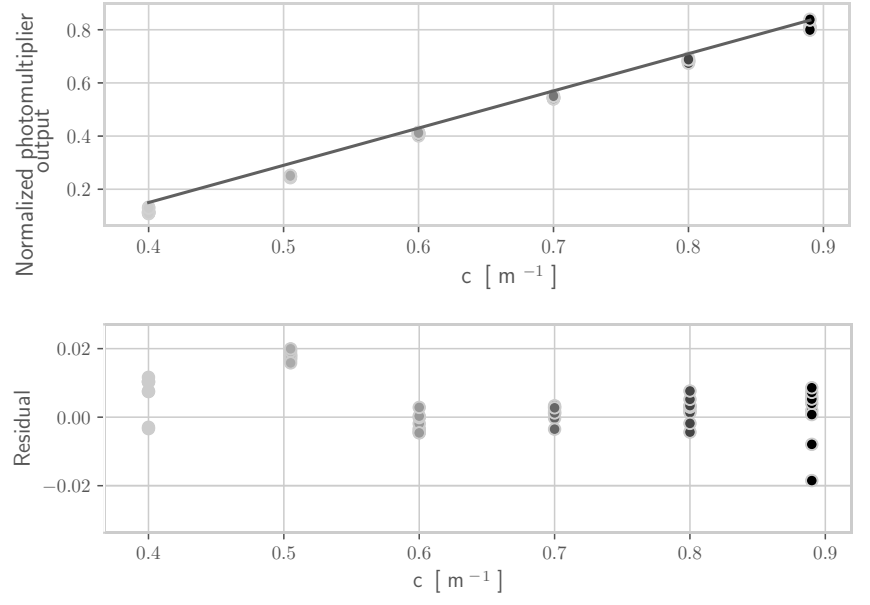

Fig. 3. (Top) Estimated linear fit of the average backscattering peak value against the total attenuation coefficient of the tank water. (Bottom) Residual errors in the estimation of the turbidity value.

is in fact sufficient to achieve very low residual error (bottom panel), on the order of $\pm 0.02 \mathrm{~m}^{-1}$ or less, which is sufficiently accurate to inform the subsequent processing steps.

\section{B. Backscattering removal}

As backscattered light from particles suspended in water can strongly corrupt the quality of the LiDAR imaging process, it becomes important to compensate for, at least, the backscattering intensity peak. To achieve this, we consider the same calibration data set employed to estimate the relationship between the total attenuation coefficient $c$ and the backscattering peak intensity. We recall that a LiDAR scan comprises a matrix of $200 \times 200$ received pulses, and that 256 samples are extracted for every channel of each pulse at a sampling interval of $0.5 \mathrm{~ns}$. We employ this data to generate an average backscattering pulse to be employed for calibration. This pulse is obtained by summing the detected light intensity samples both over all $200 \times 200$ pulses in a frame and over several frames, and by finally normalizing the pulse so that it has a maximum value of 1 . We then consider a Gamma probability density function (pdf) of the form

$$
f(x ; k, \theta)=\frac{x^{k-1} \mathrm{e}^{-x / \theta}}{\theta^{k} \Gamma(k)}, \quad x>0, k>0, \theta>0,
$$

where $\Gamma(k)$ is Euler's Gamma function, and we employ leastsquares fitting to estimate the parameters of the function that best approximate the shape of the pulse.

Before we can actually compensate backscattering, we need to create another average map that conveys the peak backscattering intensity, and that will be used to scale the Gamma function approximation. We obtain these maps from the calibration frames, by recording the average backscattering peak intensity for each of the $200 \times 200$ signals of a frame. In Fig. 4, we present three heatmaps that show, for each signal, the amplitude of the backscattering peak. The values 
$\mathrm{Rx} 1$

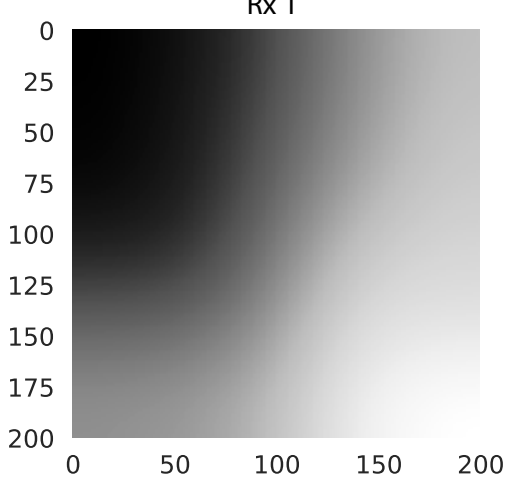

$\mathrm{R} \times 2$

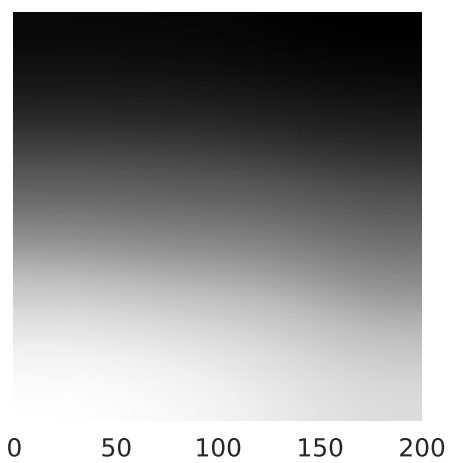

$\operatorname{Rx} 3$

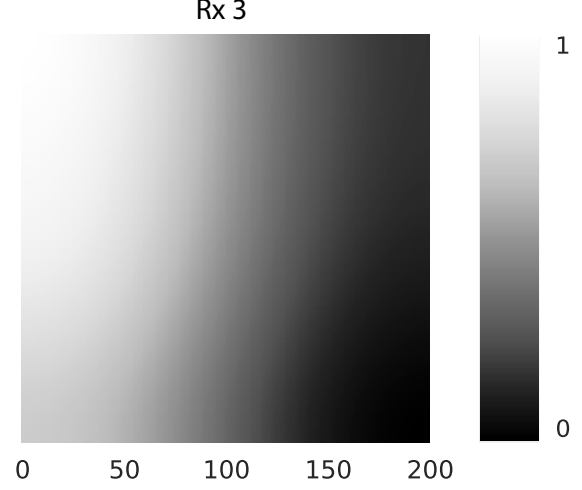

Fig. 4. Backscattering peak amplitude heatmaps for the three receivers.

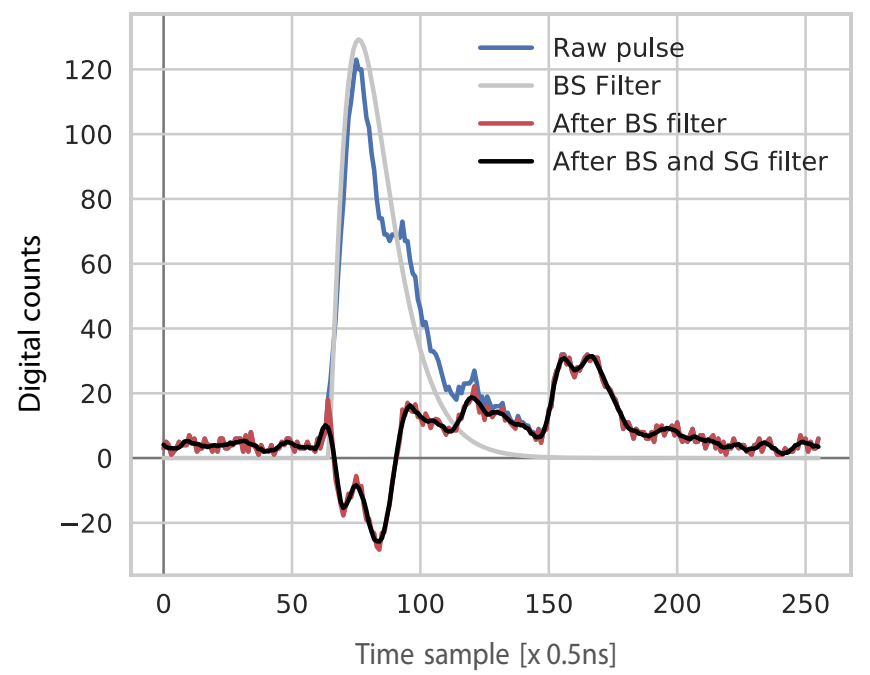

Fig. 5. Example of pulse backscattering removal based on the estimated turbidity and the backscattering model of Figs. 2 and 4.

are normalized with respect to the digital counts corresponding to the maximum expected backscattering value. Such digital counts are the output of the digitizer of the photomultiplier output, and are proportional to the instantaneous detector current, which in turn is proportional to the time-resolved scene irradiance (in $\mathrm{W} / \mathrm{m}^{2}$ ) at $638 \mathrm{~nm}$. After scaling each signal in accordance with the heatmap, the backscattering component of the signal can be subtracted in order to enhance the contrast of any other peaks reflected by a target, if present.

In Fig. 5, we provide an example of backscattering removal from light intensity signals. The blue line is the detected signal before backscattering removal, whereas the grey line is its approximation through a Gamma pdf. The red curve is obtained after the subtraction of modeled backscattering. We observe that the red curve already contains a distinguishable component related to the target, located between samples 145 and 180. In order to remove residual noise, as a last step we apply a Savitzky-Golay (SG) filter. The resulting smoothed signal is shown using a black line.

The main advantage of the above processing method is that the residual backscattering intensity is now significantly smaller that the target reflection peak, so that a simple maximum search operation suffices to identify the signal coming from the target. At the cost of a simple preliminary calibration step, this method does not require to average multiple LiDAR frames, and requires fewer LiDAR scans, besides having very low complexity.

\section{C. $2 D$ image enhancement and visualization}

In order to visualize the effect of the backscattering removal, the intensity of the target peak for each receiver is coded using a red/green/blue (RGB) color vector. Each color channel conveys the intensity of one receiver: with reference to the circles in Fig. 1, we use red for receiver 1 (to the top right of the laser when facing towards the water in the tank setup), green for receiver 2 (bottom-central position in the tank), and blue for receiver 3 (top left position). The three panels on the top of the figure refer to low turbidity conditions $\left(c=0.36 \mathrm{~m}^{-1}\right)$, whereas the three bottom panels refer to relatively high turbidity $\left(c=0.75 \mathrm{~m}^{-1}\right)$. The left panel in Fig. 6 shows the RGB coding for unprocessed signals which have not undergone any backscattering compensation. Due to the location of the receivers, the top-left corner is dominated by blue hues, the top-right corner by red hues, and the bottom side by greener hues. A pixel color turning towards white represents a high intensity detected by all receivers.

For the clear water case, the image produced by the system without any processing (Fig. 6 top-left) is clear enough for further processing. However even here backscattering removal (top-middle) improves the visibility of features towards the far side of the tank. The results are better than a manual gating procedure focusing on the target-reflected light portion (top-right). In the bottom-left panel of Fig. 6, in relatively high turbidity conditions, the shape of the fish target can be observed from the purple hues in the center of the image, whereas the near-field calibration target is barely seen along the left side of the image. After backscattering removal, we obtain the image in the bottom-middle panel. Here we observe that the fish target is the brightest element, and its characteristic biological features (e.g., the shape and the fins) are much easier to distinguish. For comparison, zeroing out the backscattering component of the received signal through 

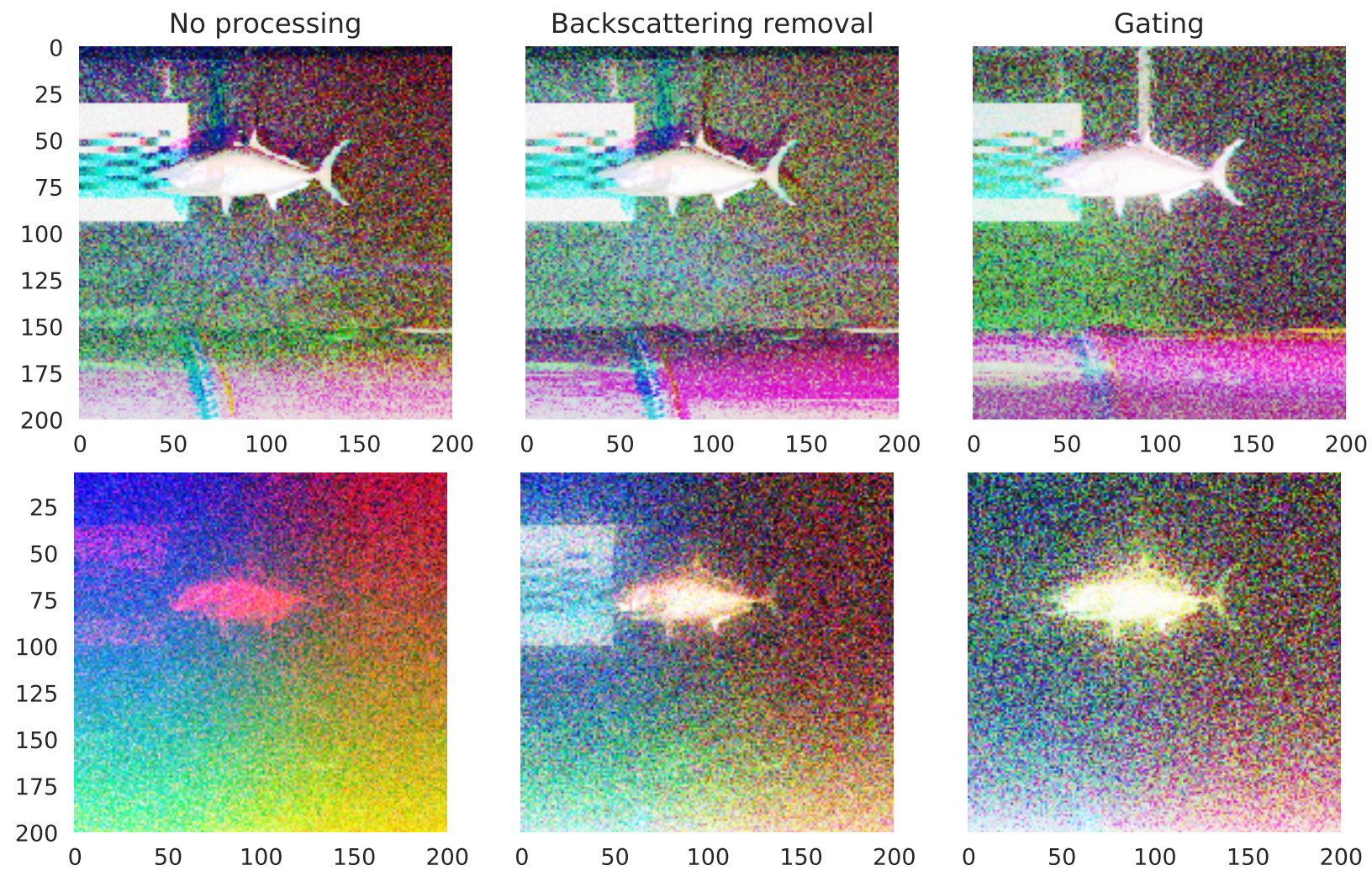

Fig. 6. Example of the RGB representation of a LiDAR image after pulse backscattering removal, compared to a simpler gating solution. (Top) $c=0.36 \mathrm{~m}^{-1}$ (Bottom) $c=0.75 \mathrm{~m}^{-1}$. The distance between the transmitter and the fish is $5.7 \mathrm{~m}\left(4.272\right.$ beam attenuation lengths at $\left.c=0.75 \mathrm{~m}^{-1}\right)$
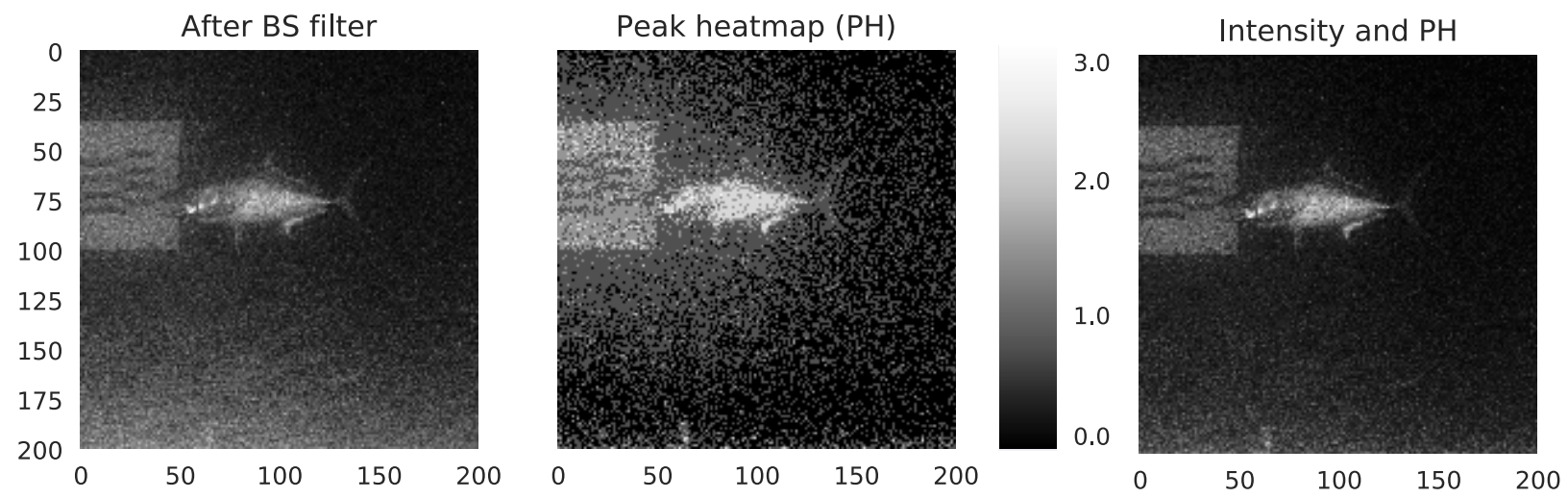

Fig. 7. Contrast enhancement for $2 \mathrm{D}$ image representation using peak heatmaps. $c=0.75 \mathrm{~m}^{-1}$.

manual gating would not be as effective in enhancing the image. An example of a gated image is shown in the bottomright panel: here, the overall image is more noisy, and the sharpness of the fish contours and biological features is greatly reduced.

We finally derive a black and white version of the target image by fusing the data from the three receivers of the LiDAR as follows. Starting from the image with backscattering removed (bottom middle panel of Fig. 6), we sum the output intensities over all channels, and normalize the intensity values to occupy the whole interval $[0,1]$. The resulting image for the $c=0.75 \mathrm{~m}^{-1}$ case is plotted in gray scale in the left panel of Fig. 7. While both the fish target and the calibration target on the left already stem clearly from the darker bottom, it is often useful to generate an image with better contrast. We do so by producing a peak heatmap where, for each pixel in the image, we count the number of peaks in the corresponding LiDAR signal. The result is shown in the middle panel, where we can observe that the target and its surroundings indeed present a higher number of intensity peaks per pixel. By performing a pixel-wise multiplication of each pixel's intensity value by the corresponding number of peaks from the heatmap, and by rescaling the resulting grey intensity values back to the interval $[0,1]$, we obtain the image in the right panel of Fig. 7. Here light intensity differences due to the shape of the fish model are amplified, and the fish contours stem more clearly out 
of a darker background. The same conclusion applies to the calibration panel on the left.

\section{Derivation of a $3 D$ target model}

Besides image processing for the enhancement of $2 \mathrm{D}$ images, it is also of interest to create a $3 \mathrm{D}$ model of the target. To do so, further processing is required to separate the light reflected by the target from backscattered or background light. To achieve this separation, we employ the Density-Based Spatial Clustering of Applications with Noise (DBSCAN) algorithm [15].

The DBSCAN algorithm is commonly used for clustering spatial data with density constraints: its run time is almost linear with the number of points to be clustered, which makes it amenable to real-time applications. Moreover, DBSCAN enables clustering without any prior knowledge about the number of clusters to be formed. As an input, the algorithm requires a definition of the meaning of "dense" clusters, conveyed by the parameters $\varepsilon$ and $N_{\text {min }}$. Specifically, $\varepsilon$ is the maximum distance between two samples that still allows both of them to belong in the same neighborhood, and $N_{\min }$ is the minimum number of samples in any cluster.

The data passed to DBSCAN is formatted as a set of samples with four features: three coordinates (to identify the location of the point in space) and an intensity value. The coordinates are expressed as sample indices, or "units:" the $\mathrm{x}$ - and $\mathrm{y}$-coordinates can take values in $0,1, \ldots, 199$ (since the scanning grid contains is $200 \times 200$ points), whereas the $\mathrm{z}$-coordinate can take values in $0,1, \ldots, 255$ (as there are 256 samples in each received signal). Since the LiDAR transmitter and receivers are synchronized, the depth of each point can be derived from the coordinates towards which the transmitter is pointed, and the round-trip time required for the light to irradiate back to each receiver. For our results, we set the parameters of DBSCAN to be $\varepsilon=4$ units and $N_{\min }=30$.

An example of the output of the clustering algorithm applied to a LiDAR frame is shown in Fig. 8. We observe that the cluster of points identifying the fish shape clearly stands out (orange cluster), along with the near-field calibration target (dark red cluster), whose border on the right side is interrupted due to the shadowing of the fish target. Spurious clusters are also present (e.g., a reflective element on the bottom of the tank

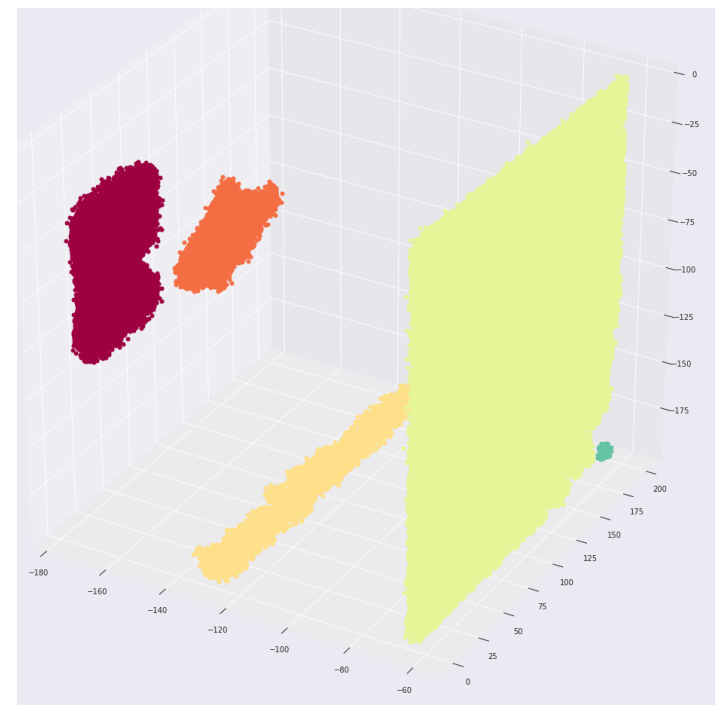

Fig. 8. Example of result obtained by applying the DBSCAN clustering algorithm to a LiDAR frame. The shape of the fish target (orange) can be clearly distinguished in the image, along with the calibration target (dark red) and with the backscattering residual (light yellow on the right).

(dark yellow) and a large planar cluster near the transmitter (light yellow, on the right of the picture) which corresponds to residual backscattering intensity that could not be completely canceled via the method described in Section III-B. Still such spurious clusters are typically well separated, so that it is possible to at least distinguish the shape and size of the fish.

Fig. 9 shows three different point clusters pertaining to the fish target, in three different turbidity conditions: low turbidity $\left(c=0.36 \mathrm{~m}^{-1}\right.$, left panel), intermediate turbidity ( $c=0.57 \mathrm{~m}^{-1}$, middle panel), and high turbidity $(c=$ $0.75 \mathrm{~m}^{-1}$, right panel). In each figure, the size of each point is proportional to the intensity of the signal received from that location, whereas the red, green and blue colors encode the receiver of the signal from which the point was estimated. As expected, in the presence of low water turbidity, several fish features can be already distinguished directly from the cloud of points. In the left panel of Fig. 9, this includes all fins, and the shape of the open mouth. As turbidity increases, the weakest reflectors of the fish (e.g., the fins) mix up
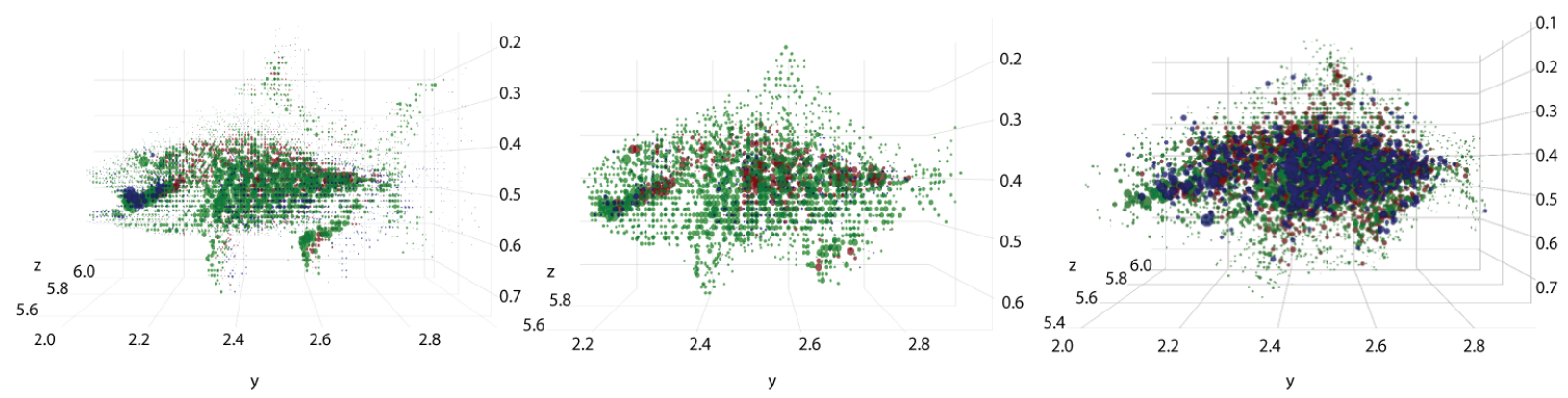

Fig. 9. Sets of points corresponding to the fish target after clustering. The color of each point corresponds to the receiver (see also Fig. 1 for reference), whereas the size of marker is proportional to intensity. The coordinates are expressed in meters. (Left) low turbidity, $c=0.36 \mathrm{~m}^{-1}$ ); (Center) medium turbidity, $\left.c=0.57 \mathrm{~m}^{-1}\right)$; (Right) high turbidity, $c=0.75 \mathrm{~m}^{-1}$ ). 


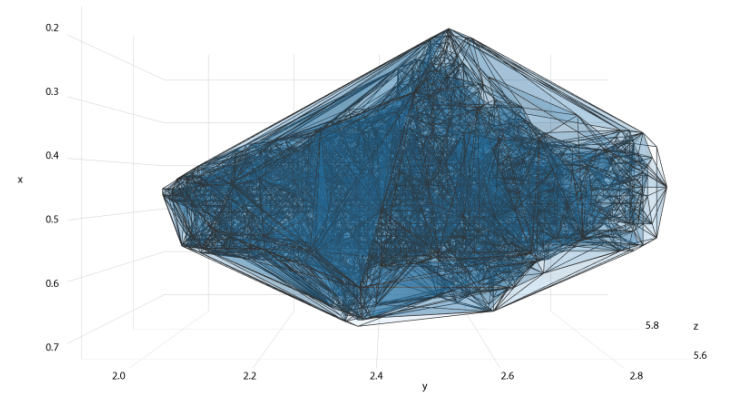

Fig. 10. Delaunay triangulation applied to the points of the identified fish cluster.

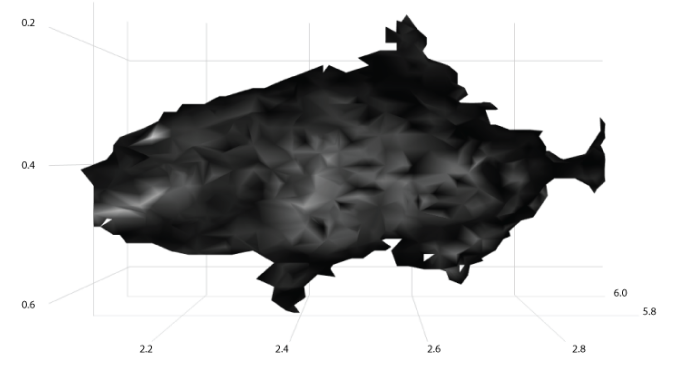

Fig. 12. $\alpha$-shape of the identified fish cluster, $c=0.57 \mathrm{~m}^{-1}$.

with backscattered light, resulting in an overall increase of the fish size, with less sharp transitions along the contour of the fish's body. In the highest turbidity conditions, the blurring of the borders becomes even worse, although it is still possible to distinguish the shape of the fish from the most reflective components of its body (in this case, the side facing the LiDAR's receivers and the caudal fin). Still, we remark that even in high turbidity conditions the backscattering compensation and the subsequent processing of the LiDAR signals make it possible to create sufficiently separated sets of points, from which the target-related points can be successfully singled out by DBSCAN.

As a last processing step, we are interested in forming a 3D model of the imaged target, which is typically useful for classification and biomass evaluation purposes. In order to achieve this, we start from the clustered samples derived from the previous step and obtain a first 3D model via Delaunay triangulation. For $c=0.36 \mathrm{~m}^{-1}$, the result is shown in Fig. 10. The plain application of the triangulation algorithm creates triangular simplices that altogether enclose the fish figure within a convex hull. However the outer simplices tend to connect much farther points, and thus have larger sides, compared to the simplices that connect the denser points of the cloud corresponding to the body and fins of the fish. This makes it possible to create an alpha-shape [16] by ruling out the simplices of the Delaunay triangulation that have a circumscribing sphere of radius greater than $\alpha$. The value of

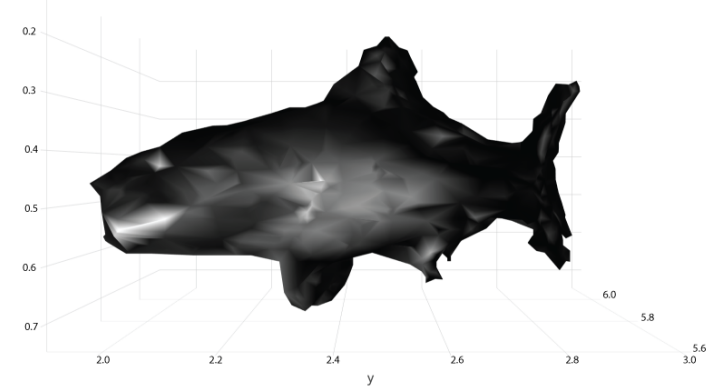

Fig. 11. $\alpha$-shape of the identified fish cluster, $c=0.36 \mathrm{~m}^{-1}$.

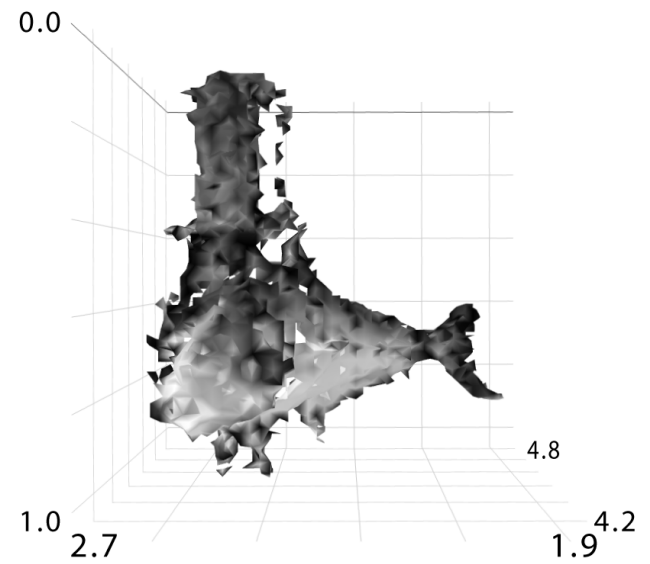

Fig. 13. $\alpha$-shape of the identified fish cluster in a more complex pose facing towards the LiDAR's receivers. In this case, the pole mount of the fish model becomes visible. $c=0.36 \mathrm{~m}^{-1}$.

$\alpha$ can be chosen to strike a balance between the accuracy of the 3D fish model and the need to achieve a connected complex of simplices. In this work, we set $\alpha=17$, which corresponds to eliminating simplices which have a circumscribed sphere of radius larger than about $6 \mathrm{~cm}$.

Fig. 11 shows the alpha-shape computed for the low turbidity point set in the left panel of Fig. 9. The shade of grey on each face encodes the intensity of the samples at the corner of the respective simplices. We observe that besides having a good model of the features of the fish, its length can be estimated quite reliably from the figure to a total head-to-tail extension of $1.14 \mathrm{~m}$. While the actual length of the fish model is $1.3 \mathrm{~m}$ the difference can be explained by the relatively low sampling frequency, which translates to a coarse depth sensing where a displacement of one sample corresponds to a length of about $11 \mathrm{~cm}$ in the tank. Part of the error is also due to the slight smoothing of protruding features, such as the fish nose and tail. In the presence of higher turbidity (e.g., as in the middle panel of Fig. 9), the alpha-shape is more noisy as can be observed in Fig. 12, and some fish details are lost after the execution of the triangulation algorithm. In any event, the shape and size of the fish are still recognizable, and the length can be measured with a sufficient degree of accuracy for biomass assessment. To conclude, we consider the imaging of the fish target rotated counter-clockwise by an angle of about $60^{\circ}$ with respect to the horizontal pose of Figs. 10 and 12 . The result of the LiDAR scan in low turbidity conditions is 
shown in Fig. 13. Interestingly, in this case the anchoring pole to which the fish target is attached is included in the fish point cluster singled out by DBSCAN, due to its greater proximity to the rest of the fish samples. Even in this case, the fish is rendered correctly, with several visible biological details, and the grey scale intensity coding reveals that the tail of the fish is farther from the LiDAR receiver than the head and pole.

\section{CONCLUSIONS}

In this paper, we presented the design of the Florida Atlantic University (FAU) Harbor Branch Oceanographic institution (HBOI)'s Unobtrusive Multistatic Serial LiDAR Imager. This LiDAR system can scan its surrounding using a low average power red laser at a wavelength of $638 \mathrm{~nm}$, which makes it non-invasive for fish vision and behavior. The LiDAR is based on inexpensive components. We presented several in-tank measurements carried out both in the absence and in the presence of a model fish target. Our results show that a simple calibration step is sufficient to estimate nearfield water turbidity and thus inform the following image processing steps. These steps include both $2 \mathrm{D}$ image contrast enhancement, and further processing to extract the LiDAR samples related to the target from the background as well as from backscattering residuals, and construct a $3 \mathrm{D}$ sensed target model. The relatively low complexity of such processing makes the system amenable to be implemented in real time.

\section{ACKNOWLEDGMENT}

The instrument development and data collection tests were supported by US Department of Energy contract DEEE0006787. The authors greatly appreciate the efforts of FAU Harbor Branch engineers Brian Ramos and Ben Metzger in developing the instrument and in conducting the experiments.

This work was sponsored in part by the European Commission's Horizon 2020 programme under grant agreement No. 773753 (SYMBIOSIS).

\section{REFERENCES}

[1] J. Karnowski, E. Hutchins, and C. Johnson, "Dolphin detection and tracking," in Proc. IEEE WACVW, Waikoloa, HI, Jan. 2015.
[2] L. Dubbs, A. G. Keeler, and T. O'Meara, "Permitting, risk and marine hydrokinetic energy development," The Electricity Journal, vol. 26, no. 10, pp. 64-74, 2013.

[3] A. W. Stoner, C. H. Ryer, S. J. Parker, P. J. Auster, and W. W. Wakefield, "Evaluating the role of fish behavior in surveys conducted with underwater vehicles," Canadian Journal of Fisheries and Aquatic Sciences, vol. 65, no. 6, pp. 1230-1243, 2008.

[4] A. E. Copping, B. Polagye, R. Suryan, S. Kramer, J. B. Saracino, and C. Smith, "Instrumentation for monitoring around marine renewable energy converters: Workshop final report," Pacific Northwest National Lab. (PNNL), Richland, WA (United States), Tech. Rep., 2014.

[5] Z. Cheng, K. Yang, J. Han, Y. Zhou, L. Sun, W. Li, and M. Xia, "Improved time-of-flight range acquisition technique in underwater lidar experiments," Applied optics, vol. 54, no. 18, pp. 5715-5725, 2015.

[6] S. P. O'Connor, L. J. Mullen, and B. Cochenour, "Underwater modulated pulse laser imaging system," Optical Engineering, vol. 53, no. 5, 2014.

[7] R. W. Lee, J. K. Nash, B. Cochenour, and L. J. Mullen, "Pulse compression techniques to improve modulated pulsed laser line scan systems," 2015.

[8] F. Dalgleish, B. Ouyang, A. Vuorenkoski, B. Ramos, G. Alsenas, B. Metzger, Z. Cao, and J. Principe, "Undersea lidar imager for unobtrusive and eye safe marine wildlife detection and classification," in Proc. MTS/IEEE OCEANS, Aberdeen, Scotland, Jun. 2017.

[9] F. M. Caimi. and F. R. Dalgleish, "Performance considerations for continuous-wave and pulsed laser line scan (LLS) imaging systems," Journal of the European Optical Society, vol. Rapid Publications 5, 2010.

[10] F. R. Dalgleish, F. M. Caimi, W. B. Britton, and A. C. F., "Improved LLS imaging performance in scattering-dominant waters," in Proc. of SPIE, vol. 7317, 2009.

[11] F. Dalgleish, A. Vuorenkoski, G. Nootz, B. Ouyang, and F. Caimi, "Experimental study into the performance impact of environmental noise on undersea pulsed laser serial imagers," Journal of Underwater Acoustics (USN), vol. 61, Oct. 2011.

[12] F. R. Dalgleish, A. K. Vuorenkoski, and B. Ouyang, "Extended-range undersea laser imaging: Current research status and a glimpse at future technologies," Marine Technology Society Journal, vol. 47, pp. 128-147, 2013.

[13] C. A. Brock, H. H. Jonsson, J. C. Wilson, J. E. Dye, D. Baumgardner, S. Borrmann, M. C. Pitts, M. T. Osborn, R. J. DeCoursey, and D. C. Woods, "Relationships between optical extinction, backscatter and aerosol surface and volume in the stratosphere following the eruption of mt. pinatubo," Geophysical research letters, vol. 20, no. 22, pp. 25552558, 1993

[14] W. Schröer, J. Köser, and F. Kuhnen, "Light-scattering in turbid fluids: The single-scattering intensity," Journal of Molecular Liquids, vol. 134, no. 1, pp. 40-48, 2007.

[15] M. Ester, H.-P. Kriegel, J. Sander, X. Xu et al., "A density-based algorithm for discovering clusters in large spatial databases with noise." in Proc. KDD, vol. 96, no. 34, Portland, Oregon, 1996, pp. 226-231.

[16] H. Edelsbrunner, D. Kirkpatrick, and R. Seidel, "On the shape of a set of points in the plane," IEEE Trans. Inf. Theory, vol. 29, no. 4, pp. $551-559,1983$ 